\title{
CLASSIFICATION OF ONE-DIMENSIONAL HYPERGROUPS
}

\author{
ALAN L. SCHWARTZ
}

(Communicated by Richard R. Goldberg)

\begin{abstract}
H, *)$ is a hypergroup if $H$ is a locally compact space and * is a binary operation with respect to which $M(H)$, the bounded Borel measures on $H$, becomes a Banach algebra with a number of additional properties so that $*$ generalizes group convolution.

The case when $H$ is a one-dimensional set, that is, a circle or an interval (possibly unbounded), includes a large number of examples: the classical group algebras $M(R)$ and $M(T)$ ( $T$ is the unit circle), the subalgebra of $M(R)$ consisting of even measures, the subalgebra of $M\left(R^{n}\right)$ consisting of rotation invariant measures, and the subalgebra of $M\left(S^{n}\right)\left(S^{n}\right.$ is the unit sphere in $R^{n+1}$ ) consisting of zonal measures. In addition to these, there are several continua of measure algebras unrelated to groups or geometry such as the measure algebras which arise in connection with Hankel transforms, ultraspherical and Jacobi polynomial series, and Sturm-Liouville expansions.

The main result of this article is a classification of the one-dimensional hypergroups. It is shown that if a certain amount of "regularity" for * is assumed, then every one-dimensional hypergroup is commutative and, up to a change of variables, must be one of the following types:

(i) the classical group algebra $M(R)$,

(ii) the classical group algebra $M(T)$,

(iii) $(H, *)$, where $H=[0, P]$ or $[0, \infty), \delta_{0}$ is an identity for $*$, and $0 \in$ $\operatorname{supp}\left(\delta_{x} * \delta_{y}\right)$ if and only if $x=y$.
\end{abstract}

1. Definitions. Let $H$ be a locally comapct space and let $M(H)$ denote the bounded Borel measures on $H$; if $\mu \in M(H), \operatorname{supp}(\mu)$ is the support of $\mu$. The unit point mass concentrated at $x$ is indicated by $\delta_{x} ; C(H)$ is the space of continuous real-valued functions on $H$; and $C_{c}(H)$ consists of all $f$ in $C(H)$ with compact support.

If $M(H)$ is a Banach algebra with multiplication $*$, then $(H, *)$ is a hypergroup if the following axioms are satisfied:

H1. If $\mu$ and $\nu$ are probability measures, then so is $\mu * \nu$.

H2. The mapping $(\mu, \nu) \rightarrow \mu * \nu$ is continuous from $M(H) \times M(H)$ into $M(H)$ where $M(H)$ is given the weak topology with respect to $C_{c}(H)$.

H3. There is an element $e \in H$ such that $\delta_{e} * \mu=\mu * \delta_{e}=\mu$ for every $\mu \in M(H)$.

H4. There is a homeomorphic mapping $x \rightarrow x^{\vee}$ of $H$ into $H$ such that $x^{\vee \vee}=x$ and $e \in \operatorname{supp}\left(\delta_{x} * \delta_{y}\right)$ if and only if $y=x^{\vee}$.

H5. For $\mu, \nu \in M(H),(\mu * \nu)^{\vee}=\nu^{\vee} * \mu^{\vee}$ where $\mu^{\vee}$ is defined for $\mu \in M(H)$ by $\int f(x) d \mu^{\vee}(x)=\int f\left(x^{\vee}\right) d \mu(x)$.

Received by the editors May 1, 1987.

1980 Mathematics Subject Classification (1985 Revision). Primary 43A10; Secondary 33A45.

The author wishes to express his gratitude to the School of Mathematical Sciences at Tel Aviv University for its hospitality during the preparation of this paper. 
H6. The mapping $(x, y) \rightarrow \operatorname{supp}\left(\delta_{x} * \delta_{y}\right)$ is continuous from $H \times H$ into the space of compact subsets of $H$ as topologized in [9].

REMARKS. 1. Property $\mathrm{H} 6$ is included for the sake of completeness, but is only required in the sense that $\operatorname{supp}\left(\delta_{s} * \delta_{t}\right)$ shrinks to $\{t\}$ as $s \rightarrow 0$.

2. The usual definition of a hypergroup is given in terms of a mapping $(x, y) \rightarrow$ $\delta_{x} * \delta_{y}$, so the entire algebra is determined by a knowledge of these products; the definition given above is equivalent to the usual one.

3. Hypergroups have many of the properties associated with the classical convolution algebras $M(G)$ when $G$ is a topological group; many of them are catalogued in $[\mathbf{4}, \mathbf{6}, \mathbf{1 1}]$ and the references given in $[\mathbf{1 1}]$.

Throughout the paper $H$ will be a one-dimensional set, that is a circle or a (possibly unbounded) real interval. The hypergroup $(H, *)$ is differentiable if whenever $f \in C_{c}(H)$ is $k$ times continuously differentiable, then $u(s, t)=\int f(u) d\left(\delta_{s} * \delta_{t}\right)(u)$ is $k$ times continuously differentiable on the interior of $H \times H$. Let $k_{\mu}$ be the largest positive integer $k$ such that for every $t$ interior to $H$

$$
M_{\mu}(s, t)=\int_{H}(r-t)^{\mu} d\left(\delta_{s} * \delta_{t}\right)(r)=O\left((s-e)^{k}\right)
$$

(the bound may depend upon $t$ ). If the relation is satisfied for no positive integer $k$, let $k_{\mu}=\infty$. Similarly define $\tilde{k}_{\mu}$ as the largest integer such that

$$
M_{\mu}^{\sim}(s, t)=\int_{H}(r-s)^{\mu} d\left(\delta_{s} * \delta_{t}\right)(r)=O\left((t-e)^{k}\right)
$$

$(H, *)$ is regular if it is differentiable and at least one of $k_{1}, k_{1}^{\sim}, k_{2}, k_{2}^{\sim}$ is finite.

Two one-dimensional hypergroups $(H, *)$ and $(K, \circ)$ are equivalent if there is a continuous monotone $\varphi \in C(H)$ such that $\varphi(H)=K$ and for every $f \in C(K)$

$$
\int_{K} f(z) d\left(\delta_{x} \circ \delta_{y}\right)(z)=\int_{H} f(\varphi(r)) d\left(\delta_{s} * \delta_{t}\right)(r)
$$

where $s=\varphi^{-1}(x)$ and $t=\varphi^{-1}(y)$. In this case we say that $\varphi$ is a domain transformation from $H$ to $K$ and that $K$ is obtained from $H$ by a change of variables. In this case the two measure algebras are isometrically isomorphic. If $(H, *)$ is differentiable and $\varphi$ is infinitely differentiable then $(K, \circ)$ is also differentiable.

2. Examples. Many classical measure algebras related to harmonic analysis and special functions are differentiable one-dimensional hypergroups. The explicit description of Examples 4-6 is too technical for presentation here and not required for this paper, so they are described breifly and sources which contain more detailed descriptions are provided.

1. The classical group algebra $M(R)$ is obtained if $H=R, \delta_{x} * \delta_{y}=\delta_{x+y} ; e=0$ and $x^{\vee}=-x$.

2. The classical group algebra $M(T)$ is obtained if $H=T=\{z \mid z$ complex, $|z|=1\}, \delta_{z} * \delta_{w}=\delta_{z w}, e=1$ and $z^{\vee}=\bar{z}=z^{-1}$.

3. The subalgebra of $M(R)$ consisting of even measures is obtained if $H=R^{+}=$ $[0, \infty), \delta_{x} * \delta_{y}=\frac{1}{2}\left[\delta_{x+y}+\delta_{|x-y|}\right], e=0$ and $x^{\vee}=x$.

4. The subalgebra of $M\left(R^{n}\right)$ consisting of rotation invariant measures may be identified with a hypergroup with domain $R^{+}, x^{\vee}=x$, and $e=0$. For $x, y>0$, $\delta_{x} * \delta_{y}$ is an absolutely continuous measure supported in ||$\left.x-y \mid, x+y\right]$. These 
hypergroups are special cases of a continuum of hypergroups related to the Hankel transform [13].

5. An example similar to the above is obtained from the algebra of zonal measures on the unit sphere in $R^{n+1}$. In this case $H=[-1,1], e=1$ and $x^{\vee}=x$. These hypergroups also belong to a continuum of hypergroups related to the Gegenbauer or ultraspherical polynomials [1 and $\mathbf{3}]$.

6. The convolutions related to Jacobi polynomials have $H, e$ and $x^{\vee}$ as in Example 5 [5].

7. Naimark's example [10, Chapter IV, 20.3; 6, 9.5]). Let $H=R^{+}$and define $\delta_{x} * \delta_{y}$ by $\delta_{0} * \delta_{x}=\delta_{x}$ and

$$
\int f(z) d\left(\delta_{x} * \delta_{y}\right)(z)=\frac{1}{\sinh x \sinh y} \int_{|x-y|}^{x+y} f(z) \sinh z d z
$$

8. Some interesting related hypergroup structure for which $H=\{0,1,2, \ldots\}$ including the duals to Examples 5 and 6 are studied in $[\mathbf{7}, \mathbf{1 3}$, and 14]. Both [7 and 14] include a characterization of hypergroups on $H=\{0,1,2, \ldots\}$ which arise from orthogonal polynomials.

3. The main result. The principal result of this article is a classification of the one-dimensional regular hypergroups.

THEOREM. Let $H$ be an interval or a circle and suppose that $(H, *)$ is a regular hypergroup. Then * must be a commutative operation and $(H, *)$ is equivalent to one of the following:

(i) $(G, *)$ where $G$ is either the circle group or the group of real numbers and * is classical group convolution on $M(G)$ (cf. Examples 1 and 2).

(ii) $(K, *)$ where $K=[0, P]$ or $K=[0, \infty), e=0$ and $x^{\vee}=x$ for every $x \in K$.

The theorem will be proved in $\S 5$. Througout the rest of this paper, $(H, *)$ will represent a one-dimensional regular hypergroup. If $H$ is an interval, it will be assumed that $e=0$ and $H \cap(0, \infty)$ is not empty, since this can be achieved by one of the domain transformations $\varphi(t)= \pm(t-e)$.

4. Generalized translation. For $f \in C(H)$ and $s, t \in H$ define

$$
T^{s} f(t)=\int_{H} f(r) d\left(\delta_{s} * \delta_{t}\right)(r)
$$

The operators $T^{s}$ are generalized translations as defined by Levitan [8] whose discussion of infinitesimal operators is adapted here. Unfortunately, the discussion in [8] excludes many of the most important examples such as 4, 5 and 6 because the assumption is made that the infinitesimal operators defined below are regular differential operators on $H$, while the infinitesimal operators associated with Examples 4, 5 and 6 are actually singular. The reader is referred to [8] for those details which do not depend on regularity and which are not included here. The $\nu$ th order infinitesimal operators of $T$ are defined for sufficiently smooth $f$ by

$$
L_{s}^{\nu} f(t)=\left.\frac{\partial^{\nu}}{\partial s^{\nu}} T^{s} f(t)\right|_{s=0}, \quad \tilde{L}_{t}^{\nu} f(s)=\left.\frac{\partial^{\nu}}{\partial t^{\nu}} T^{s} f(t)\right|_{t=0}
$$

writing $u(s, t)=T^{s} f(t)$, it follows $[\mathbf{1}$, p. 28] that $u$ satisfies the partial differential equations

$$
L_{s}^{\nu} u=\tilde{L}_{t}^{\nu} u
$$


Explicit expressions for $L_{s}^{\nu}$ are obtained with the help of the moments

$$
M_{\mu}(s, t)=\int_{H}(r-t)^{\mu} d\left(\delta_{s} * \delta_{t}\right)(r) .
$$

For each $t$ in $H, \operatorname{supp}\left(\delta_{s} * \delta_{t}\right)$ approaches $\{t\}$ as $s$ decreases to zero, so $M_{\mu}(0, t)=0$, and since $(r-t)^{\mu}$ coincides with a $C_{c}^{\infty}$ function in a compact interval containing $t$, there must be a positive integer $k_{\mu}$ and a nontrivial function $A_{\mu}$ such that for all $t$ interior to $H$

$$
M_{\mu}(s, t)=A_{\mu}(t) s^{k_{\mu}}+O\left(s^{k_{\mu}+1}\right)
$$

where $A_{\mu}$ has continuous derivatives of all orders on the interior of $H$; it also follows that $k_{2}<k_{3}, k_{4}<k_{5}, \ldots$ and $k_{2}<k_{4}<k_{6}<\cdots$ so if $\nu$ is a positive integer, there is an integer $\mu_{0} \geq \nu$ such that $\mu \geq \mu_{0}$ implies $k_{\mu}>\nu$.

Now if a sufficiently smooth $f$ is expanded in powers of $r-t$, then

(2)

$$
\begin{aligned}
u(s, t) & =\int f(r) d\left(\delta_{s} * \delta_{t}\right)(r) \\
& =\int\left[\sum_{\mu=0}^{\mu_{0}}(r-t)^{\mu} \frac{f^{(\mu)}(t)}{\mu !}+O\left((r-t)^{\mu_{0}+1}\right)\right] d\left(\delta_{s} * \delta_{t}\right)(r) \\
& =\sum_{\mu=0}^{\mu_{0}} M_{\mu}(s, t) \frac{f^{(\mu)}(t)}{\mu !}+O\left(M_{\mu_{0}+1}(s, t)\right) \\
& =\sum_{\mu=0}^{\mu_{0}} A_{\mu}(t) \frac{f^{(\mu)}(t)}{\mu !} s^{k_{\mu}}+O\left(s^{\nu+1}\right)
\end{aligned}
$$

(where $O$ depends only on $f$ and $t$ ).

By Taylor's Theorem

$$
\begin{aligned}
u(s, t) & =\left.\sum_{\lambda=0}^{\nu} \frac{\partial^{\lambda}}{\partial s^{\lambda}} u(s, t)\right|_{s=0} \frac{s^{\lambda}}{\lambda !}+O\left(s^{\nu+1}\right) \\
& =\sum_{\lambda=0}^{\nu} L_{s}^{\lambda} f(t) \frac{s^{\lambda}}{\lambda !}+O\left(s^{\nu+1}\right)
\end{aligned}
$$

which can be used together with (2) to obtain an explicit expression

$$
L_{s}^{\nu} f(t)=\nu ! \sum_{k_{\mu}=\nu} A_{\mu}(t) \frac{f^{(\mu)}(t)}{\mu !} .
$$

A similar method yields the expression

$$
\tilde{L}_{t}^{\nu} f(s)=\nu ! \sum_{k_{\tilde{\mu}}=\nu} A_{\mu}^{\sim}(s) \frac{f^{(\mu)}(s)}{\mu !}
$$

where

$$
M_{\mu}^{\sim}(s, t)=\int(r-s)^{\mu} d\left(\delta_{s} * \delta_{t}\right)(r)=A_{\mu}^{\sim}(s) t^{k_{\mu}^{\tilde{\mu}}}+O\left(t^{k_{\tilde{\mu}}^{\tilde{+}+1}}\right) .
$$

5. Proof of Main Theorem. The proof is decomposed into two cases:

Case 1. $\min \left\{k_{1}, k_{1}^{\sim}\right\}<\min \left\{k_{2}, k_{2}^{\sim}\right\}$.

Case 2. $\min \left\{k_{2}, k_{2}^{\sim}\right\} \leq \min \left\{k_{1}, k_{1}^{\sim}\right\}$.

5.1. Proof OF TheOREM IN CASE 1 FOR $H$ AN INTERVAL. 
LEMMA 1. (i) $k_{1}=k_{1}^{\sim}=1$.

(ii) $A_{1}$ and $A_{1}^{\sim}$ are bounded away from zero in a neighborhood of zero.

(iii) $A_{1}$ and $A_{1}^{\sim}$ have no zeros in $H$.

ProOF. Assume $k_{1}<\infty$. Then $A_{1}$ is a nontrivial continuous function so there is an interval $I=[a, b]$ in $H$ and $c>0$ such that $\left|A_{1}(t)\right|>c$ for $t \in I$. Choose $f \in C_{c}^{\infty}(H)$ such that $f(b)-f(a)>b-a$ and let $u(s, t)=T^{s} f(t)$. Then if $s$ is sufficiently close to zero $u(s, b)-u(s, a)>b-a$, so there is $t_{s} \in I$ such that

$$
u_{t}\left(s, t_{s}\right)>1 \text {. }
$$

To prove $k_{1}=k_{1}^{\sim}$ assume by way of contradiction that $k_{1}<k_{1}^{\sim}$. Then (1) with $\nu=k_{1}$ yields $A_{1}(t) u_{t}(s, t)=0$, but $A_{1}\left(t_{s}\right) \neq 0$, so $u_{t}\left(s, t_{s}\right)=0$ in contradiction to (3). A similar argument eliminates $k_{1}>k_{1}^{\sim}$, so the first equality in (i) is proved and (1) with $\nu=k$, takes the form

$$
A_{1}(t) u_{t}(s, t)=A_{1}^{\sim}(s) u_{s}(s, t) .
$$

Let $\varepsilon>0$; then since $f \in C_{c}^{\infty}(H), u \in C_{c}^{\infty}(H)$ there is $K>0$ such that

$$
\left|u_{s}(s, t)\right| \leq K \quad(|s| \leq \varepsilon, t \in I) .
$$

Thus if $|s| \leq \varepsilon$, equations (3) and (4) imply $c<\left|A_{1}\left(t_{s}\right) u_{t}\left(s, t_{s}\right)\right|=\left|A_{1}^{\sim}(s) u_{s}\left(s, t_{s}\right)\right|$ $\leq K\left|A_{1}^{\sim}(s)\right|$ which yields (ii) for $A_{1}^{\sim}$; the result for $A_{1}$, is obtained by symmetry.

The second equality in (i) follows because if $k_{1}>1, u_{x}(0, t)=L_{s}^{1} f(t)=0$, so $u$ satisfies $A_{1}(t) u_{t}=A_{1}^{\sim}(s) u_{s}, u(0, t)=f(t)$ and $u_{s}(0, t)=0$ which is impossible unless $f$ is constant.

Suppose that for some $s_{0}, t \in H, A_{1}^{\sim}\left(s_{0}\right)=0$ and $A_{1}(t) \neq 0$. Then equation (4) implies $u_{t}\left(s_{0}, t\right)=0$, and since $u_{t}$ is continuous, this relation holds for all $t$ in $C$, the component of the closure in $H$ of the support of $A_{1}$ which contains 0 . Thus $u\left(s_{0}, t\right)=u\left(s_{0}, 0\right)=f\left(s_{0}\right)$ for $t \in C$, whence

$$
\delta_{s} * \delta_{t}=\delta_{s} \quad\left(A_{1}^{\sim}(s)=0, t \in C\right)
$$

and similarly

$$
\delta_{s} * \delta_{t}=\delta_{t} \quad\left(A_{1}(t)=0, s \in C^{\sim}\right)
$$

where $C^{\sim}$ is analogous to $C$.

The next step is to show

$$
C=C^{\sim}=H .
$$

The homeomorphism $t \rightarrow t^{\vee}$ is necessarily monotone; suppose first that

$$
t_{1}^{\vee}<t_{2}^{\vee} \text { whenever } t_{1}<t_{2} \text {. }
$$

Let $t_{0}=\operatorname{lub} C, s_{0}=\operatorname{lub} C^{\sim}$ and $P=\operatorname{lub} H$, so $0<s_{0}, t_{0} \leq P$. It will follow that

$$
t_{0}=s_{0}=P
$$

for if $0<t_{0}<P$, then $A_{1}\left(t_{0}\right)=0$ and (7) implies $0<t_{0}^{\vee}$. Now either $t_{0}^{\vee} \leq s_{0}$ or $t_{0}^{\vee}>s_{0}$. In the first case $t_{0}^{\vee} \in C^{\sim}$, so $\delta_{t_{0}} * \delta_{t_{0}}=\delta_{t_{0}}$ by (5) which contradicts H4 since $t_{0}>0$, while in the second case $s_{0}<P$, so $A_{1}\left(s_{0}\right)=0$. Also $s_{0}^{\vee} \in C$ so $\delta_{s_{0}} * \delta_{s_{0}^{v}}=\delta_{s_{0}}$ which again yields a contradiction, so $t_{0}=P$ and (8) follows by symmetry. A similar argument establishes the equality of the greatest lower bounds of the three sets, so (6) is proved if (7) holds. The same techniques can be used $t \rightarrow t^{\vee}$ is a decreasing function.

Now if $z \neq 0$ is a zero of $A_{1}$, then $z^{\vee} \in C^{\sim}$ so $\delta_{z^{\vee}} * \delta_{z}=\delta_{z}$ which yields a contradiction, and (iii) follows. 
LEMMA 2. $A_{1}(t)=A_{1}^{\sim}(t)$ for all $t \in H$.

ProOF. The previous lemma guarantees the existence of a solution to $A_{1}^{\sim} \psi^{\prime}=$ $\lambda \psi$ which satisfies $\psi(0)=1$, so the argument in $[8$, Lemma 6.1, p. 36] applies.

By Lemma 1

$$
\varphi(t)=\int_{0}^{t}\left[A_{1}(r)\right]^{-1} d r \quad(t \in H)
$$

is a homeomorphism from $H$ to an interval $K$. Thus $\varphi$ is a domain transformation from $(H, *)$ to a hypergroup $(K, \circ)$ where $\circ$ is defined by

$$
\int_{K} f(z) d\left(\delta_{x} \circ \delta_{y}\right)(t)=\int_{H} f(\varphi(r)) d\left(\delta_{s} * \delta_{t}\right)(r) .
$$

If $x=\varphi(s), y=\varphi(t), F(x)=f(s), U(x, y)=u(s, t)=T^{s} f(t)$, then $U$ satisifes

$$
\frac{\partial U}{\partial x}=\frac{\partial U}{\partial y}, \quad U(0, y)=F(y)
$$

so if $x, y, x+y$ all belong to $K$

$$
U(x, y)=F(x+y)
$$

or equivalently

$$
\delta_{x} \circ \delta_{y}=\delta_{x+y}
$$

Since $(K, \circ)$ is equivalent to $(H, *)$ it will be no loss of generality to assume for the next two lemmas that they are identical, so if $f$ is sufficiently smooth and $u(s, t)=T^{s} f(t)$, then $u$ satisfies

$$
u_{s}=u_{t}, \quad u(0, t)=f(t)
$$

and

$$
u(s, t)=f(s+t) \quad \text { and } \quad \delta_{s} * \delta_{t}=\delta_{s+t} \quad(s, t, s+t \in H) .
$$

Thus if $H=R,(H, *)$ is exactly the classic group algebra $M(R)$. The following two lemmas show this must be the case.

A measure $m \in M(H)$ is called Haar measure if $m * \delta_{t}=\delta_{t} * m=m$ for every $t \in H$.

LEMMA 3. $(H, *)$ is a commutative hypergroup with a Haar measure $m ; \operatorname{supp}(m)$ $=H$ and $m^{\vee}=m$.

PrOOF. Let $u$ be as above; then $u$ is constant on the characteristic lines, $s+t=$ const. of (9). So $u(s, t)=u(t, s)$ and $(H, *)$ is a commutative hypergroup. The lemma follows since every commutative hypergroup has a unique (up to multiplicative constant) Haar measure. The last two assertions are always true for Haar measures (cf. $[\mathbf{4}, \mathbf{1 5}])$.

LEMMA 4. If $H$ is an interval, then $H=R$.

ProOF. Let $\rho(t)=t+t^{\vee}$; then $\rho$ is continuous on $H$ and if $\rho(t) \in H$ then (10) and H4 imply $\rho(t)=0$. Now let $t_{0}$ be a positive number in $H$ and choose $\varepsilon$, $0<\varepsilon<t_{0} / 2$, so small that $0<t<\varepsilon$ implies $\left|t^{\vee}\right|<t_{0} / 2$. In this case $t^{\vee}<\rho(t)<t_{0}$ so $\rho(t) \in H$, hence $\rho(t)=0$ and $t^{\vee}=-t$ for $0 \leq t<\varepsilon$. It follows that $(-\varepsilon, \varepsilon) \subset H$. 
Next, $\rho(t)=0$ for all $t \in H$, for if not let $t_{0}=\operatorname{lub}\{t \mid \rho=0$ on $[0, t)\}<\operatorname{lub} H$. It is possible to choose $t_{1} \in H$ such that $t_{1}>t_{0}$ and so that $\left|\rho\left(t_{1}\right)\right|=\left|\rho\left(t_{1}\right)-\rho\left(t_{0}\right)\right|<\varepsilon$. Hence $\rho\left(t_{1}\right) \in H$ so $\rho\left(t_{1}\right)=0$ which contradicts the upper bound property of $t_{0}$.

Thus $t^{\vee}=-t$ for all $t \in H$, and $H$ is a symmetric interval.

Suppose by way of contradiction to the lemma that $P=\operatorname{lub} H<\infty$; then

$$
H=[-P, P] \text {. }
$$

For if $Q=P / 2$ and $f \in C_{c}(-P, P)$, then $\int f d\left(\delta_{Q} * \delta_{Q}\right)=\lim _{t \rightarrow Q-} \int f d\left(\delta_{t} * \delta_{t}\right)=$ $\lim _{t \rightarrow Q-} f(2 t)=0$. Hence $\delta_{Q} * \delta_{Q}$ is a probability measure on $H$ with no support in $(-P, P)$. Since the only possibilities for $H$ are $(-P, P)$ and $[-P, P]$, the former is eliminated and (11) holds.

Now let $f \in C_{c}^{+}(H)$ with $f(t)=0$ for $t \leq 0$. Let $u(s, t)=T^{s} f(t)$; then

$$
\int_{0}^{P} f(t) d m(t)=\int_{-P}^{P} u(P, t) d m(t)=\int_{-P}^{0} f(P+t) d m(t)+\int_{0}^{P} u(P, t) d m(t),
$$

and since $u(P, t) \geq 0$ for all $t$

$$
\int_{0}^{P} f(t) d m(t) \geq \int_{-P}^{0} f(P+t) d m(t) .
$$

If now $g(t)=f(P-t)$ for $0 \leq t \leq P$ and $g(t)=0$ for $t \leq 0$, then (12) must hold for $g$ in place of $f$ :

$$
\int_{0}^{P} g(t) d m(t) \geq \int_{-P}^{0} g(P+t) d m(t) .
$$

But from Lemma $3, m^{\vee}=m$, so $t$ can be replaced by $t^{\vee}=-t$ to obtain

$$
\int_{-P}^{0} g(-t) d m(t) \geq \int_{0}^{P} g(P-t) d m(t)
$$

Finally, using the definition of $g$

$$
\int_{-P}^{0} f(P+t) d m(t) \geq \int_{0}^{P} f(t) d m(t)
$$

which is the reverse of inequality $(12)$, so $u(P, t)=0$ a.e. $(d m)$. But $\operatorname{supp}(m)=H$ (Lemma 3) so $u(P, t)=0$ a.e. $(d m)$ in $[0, P]$. Since $u$ is continuous by $\mathrm{H} 2, f(P)=$ $u(P, 0)=0$. But $f$ can take any value at $P$, which yields a contradiction.

5.2. Proof OF TheOREM IN CASE 1 WheN $H$ IS A CIRCle. In the case when $H$ is a circle there is no loss in assuming that $H$ is the unit circle.

Let $f \in C(H)$ and $u(s, t)=T^{s} f(t)$. Then $f, A_{1}$ and $A_{1}^{\sim}$ can be identified with periodic functions on $R$ with period $2 \pi$ and $u$ can be identified with a function on $R \times R$ with period $2 \pi$ in each of the variables. Thus with a slight abuse of notation, Lemmas 1,2 and the arguments above yield functions $F(y)$ and $U(x, y)$ which are defined for all $x, y \in R$ with period $P=\varphi(2 \pi)$ and $U(x, y)=F(x+y)$ for all $x, y \in R$, hence $(H, *)$ is equivalent to the classical group algebra $M(T)$.

5.3. Proof OF THEOREM IN CASE 2. Assume that $k_{2} \leq k_{2}^{\sim}$; the proof is contained in the following lemmas. 
LEMMA 5. If $k_{2} \geq 2$ then $L_{s}^{1}=0$.

PROOF. The support of $\delta_{s} * \delta_{t}$ shrinks to $\{t\}$ as $s$ decreases to zero, so $\nu>2$ implies $M_{\nu}(s, t)=o\left(M_{2}(s, t)\right)$, hence $k_{\nu}>2$ if $\nu>2$, but $k_{1} \geq k_{2} \geq 2$ so the coefficient of $s$ in the expression (2) is zero, whence $L_{s}^{1}=0$.

LEMMA 6. $H \subset[0, \infty)$.

PrOOF. The cases $k_{2}=1$ and $k_{2} \geq 2$ are discussed separately.

In the first case there is $t_{0}$ such that $A_{2}\left(t_{0}\right) \neq 0$. Now

$$
A_{2}\left(t_{0}\right)=\lim _{s \rightarrow 0+} s^{-1} M_{2}\left(s, t_{0}\right),
$$

so $A_{2}\left(t_{0}\right)>0$ because $M_{2}\left(s, t_{0}\right) \geq 0$. If the lemma is false, then it must also be the case that $A_{2}\left(t_{0}\right)=\lim _{s \rightarrow 0-} s^{-1} M_{2}\left(s, t_{0}\right) \leq 0$ which is a contradiction.

In the second case, assume by way of contradiction that 0 is interior to $H$ and choose $f \in C_{c}^{\infty}$ satisfying

$$
f^{\prime}(0)=1 .
$$

Let $u(s, t)=T^{s} f(t)$. Since 0 is a two-sided identity, $u(s, 0)=f(s)$ and since 0 is interior to $H, u$ is differentiable at $(0,0)$. From Lemma $5, u_{s}(0, t)=0$; this shows $f^{\prime}(0)=u_{s}(0,0)=0$ contradicting $(13)$ and proving the lemma.

LEMMA 7. $s^{\vee}=s$ for every $s$ in $H$.

PROOF. Let $r(s)=s^{\vee}$. Then $r$ is a monotone increasing function because of Lemma 6. If for some $s \in H, s \leq s^{\vee}$ then $r(s) \leq r\left(s^{\vee}\right)$ or equivalently $s^{\vee} \leq s$ so $s=s^{\vee}$. The same argument applies if $s^{\vee} \geq s$ so the lemma follows.

LEMMA 8. $(H, *)$ is commutative.

ProOF. By Lemma 7 and H5, $\delta_{s} * \delta_{t}=\delta_{s^{\vee}} * \delta_{t \vee}=\left(\delta_{t} * \delta_{s}\right)^{\vee}=\delta_{t} * \delta_{s}$ (as in [6, Theorem 9.1.A]).

The proof for this case is completed by observing that Lemma 6 limits the possibilities for $H$ to $[0, \infty),[0, P]$ and $[0, P)$ and the first and third are equivalent by any domain transformation $\varphi:[0, P) \rightarrow[0, \infty)$.

\section{REFERENCES}

1. W. C. Connett and A. L. Schwartz, The theory of ultraspherical multipliers, Mem. Amer. Math. Soc. 183 (1977).

2. __ Convolution structures for eigenfunction expansions arising from regular Sturm-Liouville problems, Anniversary Volume on Functional Analysis and Approximation, Birkhäuser, Basel, 1984, pp. 437-447.

3. C. F. Dunkl, Operators and harmonic analysis on the sphere, Trans. Amer. Math. Soc. 125 (1966), 250-263.

4. __ The measure algebra of a locally compact hypergroup, Trans. Amer. Math. Soc. 179 (1973), 331-348.

5. G. Gasper, Banach algebras for Jacobi series and positivity of a kernel, Ann. of Math. (2) 95 (1972), 261-280.

6. R. I. Jewett, Spaces with an abstract convolution of measures, Adv. in Math. 18 (1975), 1-101.

7. R. Lasser, Orthogonal polynomials and hypergroups, Rend. Mat. (7) 3 (1983), 185-209.

8. B. M. Levitan, Generalized translation operators, Israel Program for Scientific Translations, Jerusalem, 1964.

9. E. Michael, Topologies on spaces of subsets, Trans. Amer. Math. Soc. 71 (1951), 152-182. 
10. M. A. Naimark, Normed rings, Noordhoff, Groningen, 1964.

11. K. A. Ross, Hypergroups and centers of measure algebras, Ist. Naz. Alta Mat. (Symposia Math.) 22 (1977), 189-203.

12. _ Centers of hypergroups, Trans. Amer. Math. Soc. 243 (1978), 251-269.

13. A. Schwartz, The structure of the algebra of Hankel transforms and the algebra of Hankel-Stieltjes transforms, Canad. J. Math. 23 (1971), 236-246.

14. Generalized convolutions and positive definite functions associated with general orthogonal series, Pacific J. Math. 55 (1974), 565-582.

15. _,$l^{1}$-convolution algebras: representation and factorization, Z. Wahrsch. Verw. Gebiete 41 (1977), 161-176.

16. R. Spector, Mesures invariantes sur les hypergroupes, Trans. Amer. Math. Soc. 239 (1978), 147-165.

Department of Mathematics, University of Missouri, St. Louis, Missouri 63121 